\title{
Direct Vapor-Phase Bromination of Multiwall Carbon Nanotubes
}

\author{
Ilya Mazov, ${ }^{1}$ Dmitry Krasnikov, ${ }^{1,2}$ Andrey Stadnichenko, ${ }^{1}$ Vladimir Kuznetsov, ${ }^{1}$ \\ Anatoly Romanenko, ${ }^{2,3}$ Olga Anikeeva, ${ }^{2,3}$ and Evgeniy Tkachev ${ }^{3}$ \\ ${ }^{1}$ Department of Physical Methods of Research, Boreskov Institute of Catalysis, Lavrentieva Avenue 5, Novosibirsk 630090, Russia \\ ${ }^{2}$ Department of Thermodynamic Studies, Novosibirsk State University, Pirogova street 2, Novosibirsk 630090, Russia \\ ${ }^{3}$ Department of Physics, Nikolaev Institute of Inorganic Chemistry, Lavrentieva Avenue 5, Novosibirsk 630090, Russia
}

Correspondence should be addressed to Ilya Mazov, ilya.mazov@gmail.com

Received 29 November 2011; Accepted 20 March 2012

Academic Editor: Angaiah Subramania

Copyright (c) 2012 Ilya Mazov et al. This is an open access article distributed under the Creative Commons Attribution License, which permits unrestricted use, distribution, and reproduction in any medium, provided the original work is properly cited.

\begin{abstract}
We present the simple procedure of the vapor-phase bromination of multiwall carbon nanotubes (MWNTs) at moderate temperatures. MWNTs with average diameter $9 \pm 3 \mathrm{~nm}$ were treated with $\mathrm{Br}_{2}$ vapors at $250^{\circ} \mathrm{C}$ to produce Br-functionalized product. Transmission electron microscopy analysis was used to prove low damage of MWNT walls during bromination. X-ray photoelectron spectroscopy (XPS) and differential thermal analysis (DTA) were used to investigate chemical composition of the surface of initial and brominated nanotubes. The experimental results show that the structure of MWNTs is not affected by the bromination process and the total amount of Br-containing surface functions reaches $2.5 \mathrm{wt}$. \%. Electrophysical properties of initial and brominated MWNTs were investigated showing decrease of conductivity for functionalized sample. Possible mechanism of the vapor-phase bromination via surface defects and oxygen-containing functional groups was proposed according to data obtained. Additional experiments with bromination of annealed low-defected MWNTs were performed giving Br content a low as $0.75 \mathrm{wt}$. \% proving this hypothesis.
\end{abstract}

\section{Introduction}

Carbon nanotubes (CNTs) attract great attention in the last decade due to their remarkable mechanical, optical, and electrical properties. Usage of CNTs is strongly limited due to their high chemical inertness and insolubility in conventional organic solvents, thus preventing chemical interaction with other organic species or matrices of composite materials. Chemical functionalization of the surface of CNTs is important step for modification and fine tuning of their properties by enhancing of compatibility with other materials, grafting of various functional groups, and so forth $[1,2]$.

One of the most promising methods of CNT functionalization is introduction of halogen atoms of the CNT surface. Carbon-halogen bond can be easily modified using conventional methods of organic chemistry-such as reduction, alkylation, and hydroxylation. Due to relatively high chemical inertness of CNT surface, special technique should be used in order to react halogens with nanotubes, especially for low-reactive bromine. At the same time, bromination of CNTs is more preferable as compared with more easy fluorination or chlorination due to higher reactivity of $\mathrm{C}-\mathrm{Br}$ bond in further functionalization steps.

Several approaches were used for functionalization of carbon nanomaterials with halogens-for example, microwave- and plasma-assisted halogenation [3-5], radiationinduced chlorination and bromination $[6,7]$, bromination with Lewis acids [8], usage of liquid bromine or vapors at room temperature [9-11], or electrochemically generated $\mathrm{Br}_{2}$ and $\mathrm{Cl}_{2}$ [12]. Almost all of these methods require usage of special techniques or experimental methods. Here, we report simple way of synthesis of Br-modified multiwall carbon nanotubes produced by direct treatment in bromine vapors at elevated temperature. Bromine is covalently linked with the surface of carbon nanotubes with total $\mathrm{Br}$ amount 0.5 at. $\%$ or 2.5 wt. $\%$.

\section{Experimental}

Initial MWNTs with specific surface area $\sim 330 \pm 15 \mathrm{~m}^{2} / \mathrm{g}$ were prepared by $\mathrm{C}_{2} \mathrm{H}_{4}$ decomposition over FeCo catalyst at $700^{\circ} \mathrm{C}$ and were purified by reflux with $\mathrm{HCl}$. Mean diameter 


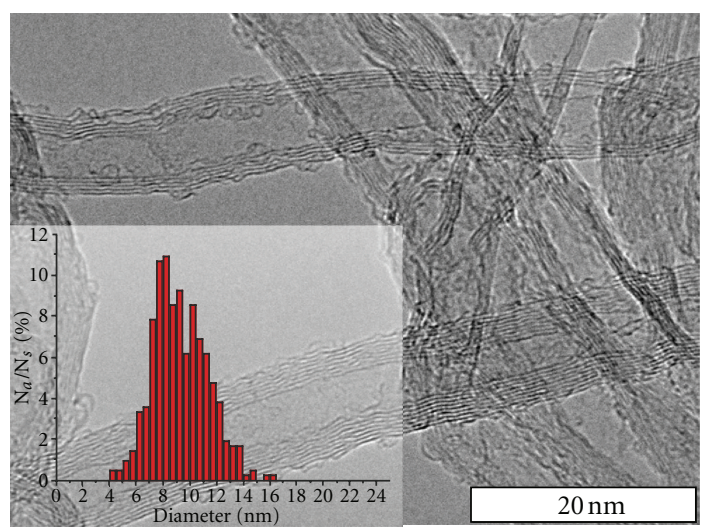

(a)

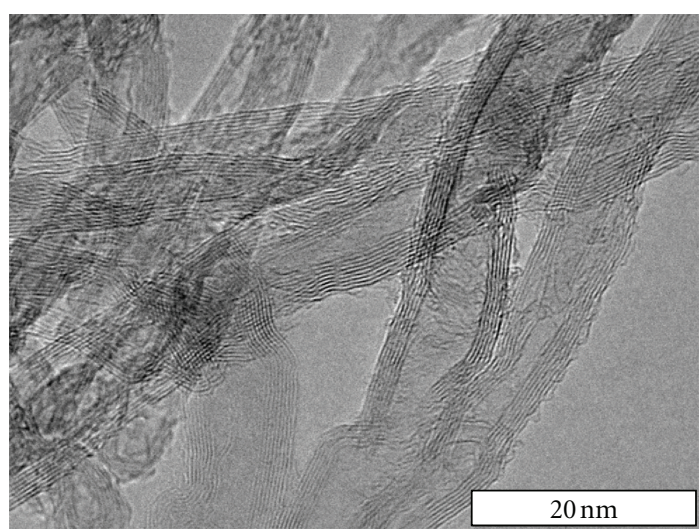

(b)

FIGURE 1: (a) High resolution TEM image of initial MWNTs with corresponding diameter distribution; (b) high resolution TEM image of the brominated sample, revealing no significant structure changes in nanotubes.

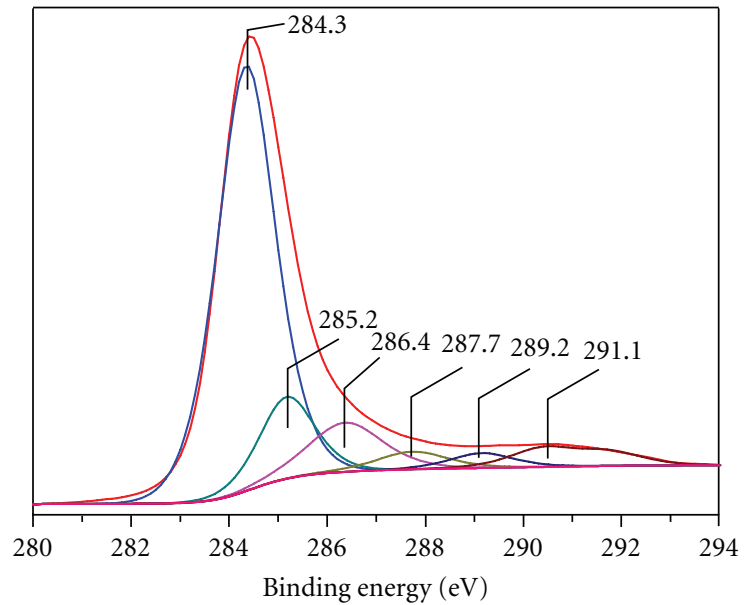

FIGURE 2: XPS C1s spectrum for both initial and brominated samples and its decomposition on individual peaks.

of MWNTs was statistically measured using TEM data and was $9 \pm 3 \mathrm{~nm}$. Bromination was carried out at $250^{\circ} \mathrm{C}$ in an electrically heated glass reactor, connected to the flask with distilled bromine kept at $80^{\circ} \mathrm{C}$. MWNTs were loaded in hot reactor filled with bromine vapors. After treatment of MWNTs with $\mathrm{Br}_{2}$ vapor, the reactor was washed with pure Ar flow and cooled to room temperature. Nanotubes were unloaded from reaction vessel and dried in vacuum $\left(10^{-2}\right.$ torr $)$ at $80^{\circ} \mathrm{C}$ overnight. Bromine-treated MWNTs $\left(\mathrm{MWNT}^{\mathrm{Br}}\right)$ and pristine MWNTs (MWNT ${ }^{\mathrm{ini}}$ ) were investigated using TEM, DTA, and XPS methods.

Additional comparative experiments by the bromination of nanotubes annealed at high temperature in pure Ar flow $\left(2800^{\circ} \mathrm{C}\right.$, see [13] for details) were performed. Annealed nanotubes possess perfect structure of graphene sheets and very low amount of structural and topological defects; thus this sample was used as comparative sample for elucidation of bromination mechanism.

\section{Results and Discussion}

Structure and morphology of initial and brominated MWNTs were investigated using scanning and transmission electron microscopy (see Figures 1(a) and 1(b)). No significant changes were observed for brominated sample, thus indicating that bromine treatment at moderate temperatures does not inflict damages in nanotube structure. EDX analysis was performed for agglomerates of nanotubes revealing 1.41.6 at. \% of bromine.

Chemical composition of initial and brominated nanotubes was investigated using XPS technique. XPS survey spectra for $\mathrm{MWNT}^{\mathrm{ini}}$ and $\mathrm{MWNT}^{\mathrm{Br}}$ samples are shown of Figure 2. Spectrum of initial nanotubes shows peaks only from C1s $(284.4 \mathrm{eV})$ and $\mathrm{O} 1 \mathrm{~s}(532.3 \mathrm{eV})$ with oxygen content $\sim 1.35$ at. \%. Spectrum of brominated nanotubes reveals peaks from C1s, O1s, Br3d (70.1 eV), Br3p (183.7 eV) with total oxygen and bromine content $\sim 1.28$ at. $\%$ and 0.41 at. $\%$, correspondingly. Note that total oxygen content decreases after bromination in contrast to observed elsewhere [3] for the process of CNT bromination in water media by microwaves. Slight decrease of oxygen content can be attributed to the substitution of oxygen atoms in carboxylic functional groups by bromine with no parallel reactions such as Br substitution by water due to nucleophilic mechanism.

High-resolution XPS spectrum of C1s shown on Figure 2 was fitted using Gauss and Lorentz peaks and DoniachSunjic curve deconvolution [14]. Peak form analysis was performed after Shirley background subtraction [15]. Following main components are observed: (1) main peak from $s p^{2}$-hybridized $\mathrm{C}$ atoms (main grapheme-like structure of the nanotube) located at $284.4 \mathrm{eV}$ (80.5 at. \%) with corresponding shake-up peak at $290.7 \mathrm{eV}$ (6.0 at. \%), (2) signal from $s p^{3}$-hybridized carbon atoms at $285.1 \mathrm{eV}$ (3.1 at. \%), (3) peak from carbon atoms in $\mathrm{C}-\mathrm{O}$ or $\mathrm{C}-\mathrm{Br}$ functional groups located at $286.2 \mathrm{eV}$ (3.7 at. \%), and (4) peak from carbonyl and carboxyl functions at $287.8 \mathrm{eV}$ (6.7 at. \%). Note that vapor-phase bromination of MWNTs does not result in any significant changes of the form and intensity of $\mathrm{C} 1 \mathrm{~s}$ line, which may be attributed to low structural changes 


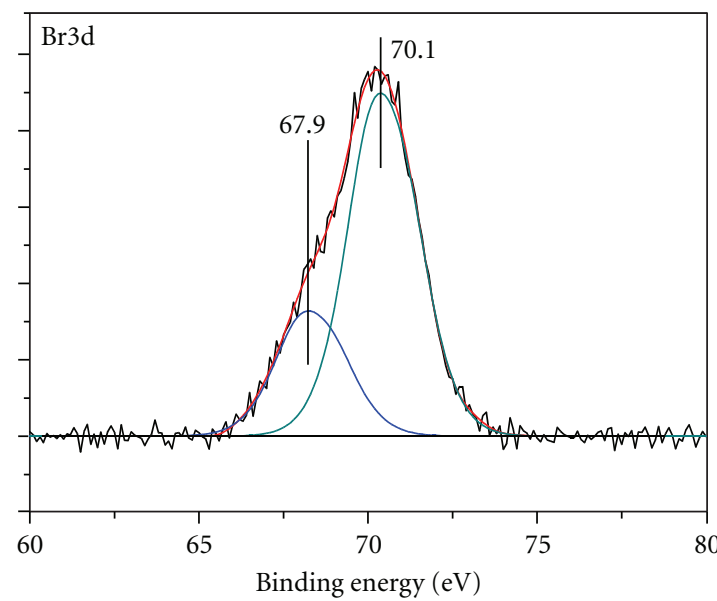

(a)

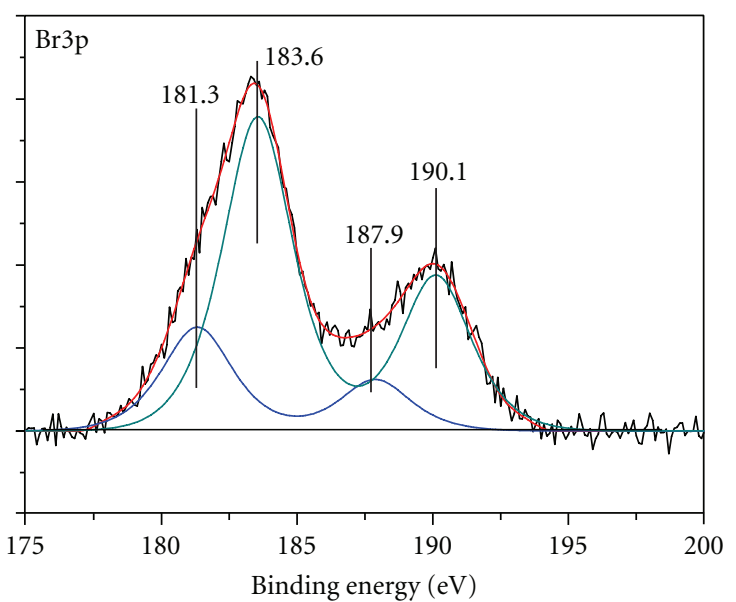

(b)

Figure 3: Decomposition of Br3d line (a) and Br3p line (b) for $\mathrm{MWNT}^{\mathrm{Br}}$ sample.

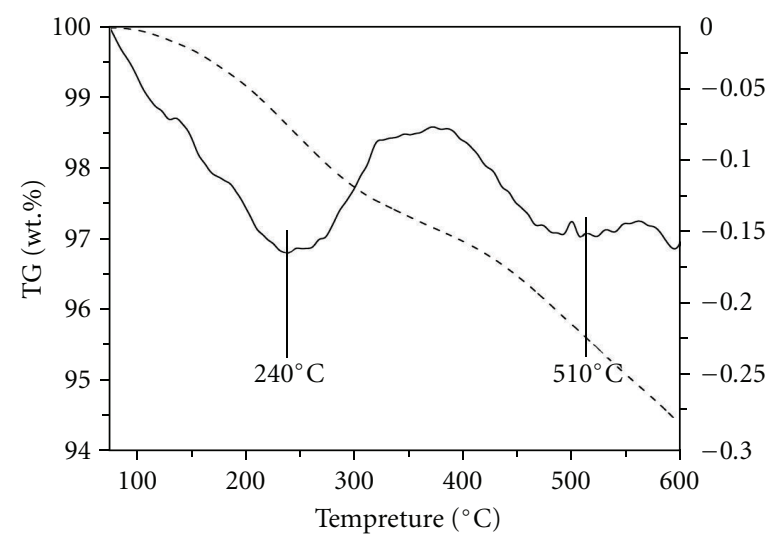

(a)

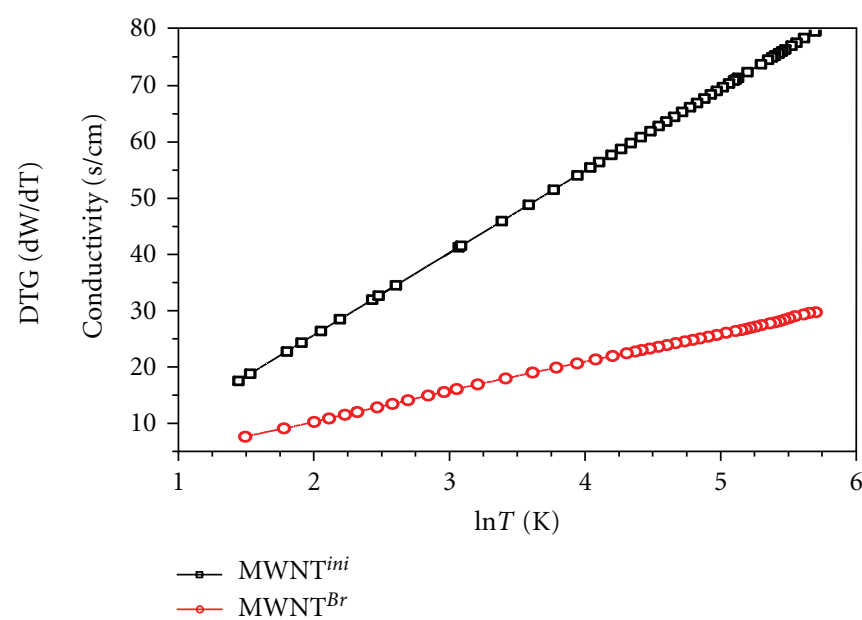

(b)

FIGURE 4: (a) Thermal analysis data for $\mathrm{MWNT}^{\mathrm{Br}}$ sample in nitrogen atmosphere at $10 \mathrm{~K} / \mathrm{min}$ (solid line represents differential thermal data, dashed line represents thermogravimetric data); (b) temperature dependencies of electrical conductivity for initial and brominated MWNTs.

of nanotubes and substitution mechanism of bromination through carboxylic surface groups (peak 3 at $286.4 \mathrm{eV}$ ), which corresponds to TEM data on the low modification of CNT structure.

High-resolution XPS spectra for $\mathrm{Br} 3 \mathrm{~d}$ and $\mathrm{Br} 3 \mathrm{p}$ lines are shown in Figure 3. Br3d spectrum (Figure 3(a)) is composed of two components with energies typical for covalently bonded $\mathrm{Br}$ atoms (70.1 eV, C-Br bonds) and negatively charged $\mathrm{Br}^{-}$species $(67.9 \mathrm{eV})$, which are most probably absorbed bromine anions of bromine salts of metal catalyst residues. The ratio between $\mathrm{C}-\mathrm{Br}$ and $\mathrm{Br}^{-}$components is near to $3: 1$. The Br3p spectrum shown on Figure 3(b) is composed of two main lines ( $\mathrm{Br} 3 \mathrm{p} 1 / 2$ and $\mathrm{Br} 3 \mathrm{p} \mathrm{3/2}$ ), each one of them can be decomposed to two components, corresponding to $\mathrm{C}-\mathrm{Br}$ bonds and $\mathrm{Br}^{-}$species on the surface of nanotubes.

Differential thermal analysis data for $\mathrm{MWNT}^{\mathrm{Br}}$ in nitrogen atmosphere are shown on Figure 4(a). First decomposition of surface species proceeds between 100 and $350^{\circ} \mathrm{C}$ with maximum decomposition temperature at $\sim 240^{\circ} \mathrm{C}$. This value is higher than temperature of desorption of physically absorbed $\mathrm{Br}_{2}$, which was most probably removed during vacuum treatment after bromination and is close to that observed elsewhere for microwave-brominated DWNTs [3] and can be associated to decomposition of $\mathrm{C}-\mathrm{Br}$ bonds. The second decomposition step occurs at $400-600^{\circ} \mathrm{C}$ with major peak at $\sim 510^{\circ} \mathrm{C}$, corresponding to possible dehydrobromination of functionalized nanotubes. Similar TGA 


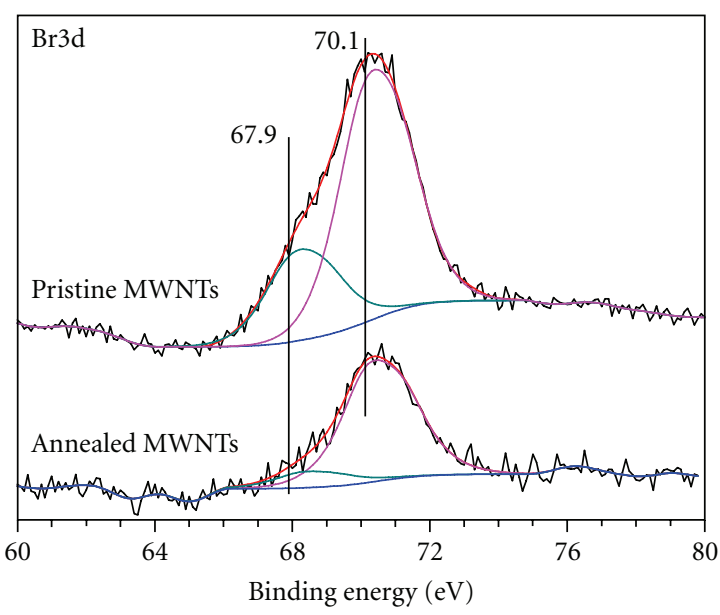

(a)

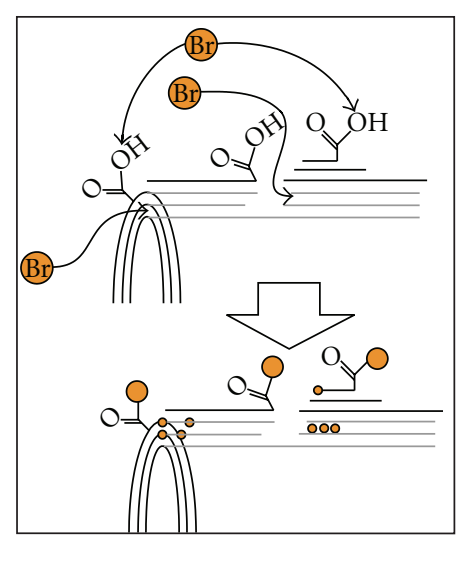

(b)

FIGURE 5: (a) High-resolution spectrum (Br3d) for initial and annealed carbon nanotubes. (b) Schematic of the Br interaction with surface groups of carbon nanotube.

behavior of brominated nanotubes was observed elsewhere and was linked to double-step dehydrobromination process with formation of allylic species on the MWNT surface [16].

According to TGA data total weight loss due to decomposition and desorption of surface species from brominated sample of nanotubes in close to $5.5 \mathrm{wt}$. \% which is reasonably close to the value of $\mathrm{Br}$ content determined by means of XPS technique $(2.5 \mathrm{wt} . \%)$ taking into consideration that desorption may proceed not only for individual bromine atoms but for $\mathrm{C}-\mathrm{Br}$-containing clusters, increasing total weight loss. Thus density of Br-containing species on the surface of MWNTs can be estimated as $0.6-0.7 \mathrm{~nm}^{-2}$. This value is close to the surface density of carboxylic groups for initial nanotubes determined by XPS and Boehm acid-base titration (not shown here).

Electrical conductivity measurements were performed for both initial and brominated samples. MWNT powder was pressed in the glass ampoule with silver contacts until the value of conductivity reached saturation. Electrophysical parameters were measured by four-probe technique successfully used earlier for investigation of various nanocarbon materials [17]. Temperature dependencies of electrical conductivity for initial and brominated sample are shown on Figure 4(b). Bromination of carbon nanotubes results in significant decrease of the electrical conductivity-sample MWNT $^{\text {ini }}$ shows conductivity value $20-80 \mathrm{~s} / \mathrm{cm}$ depending on the temperature, and the sample MWNT ${ }^{\mathrm{Br}}$ shows $\sigma$ value in the range $7-25 \mathrm{~s} / \mathrm{cm}$ (Figure $4(\mathrm{~b})$ ).

Bromine atoms act as acceptors of electrons resulting in change of the density of current carriers in surface layers of the nanotube thus decreasing electrical conductivity of the sample. Moreover, the slope of the temperature dependence curves changes with functionalization of the surface which is attributed to the changes in the mechanism of electrical conductivity. Untreated nanotubes possess logarithmic temperature dependence corresponding to two-dimensional quantum corrections to conductivity (see [18] for details), and brominated MWNTs show exponential temperature dependence of conductivity which can be described within theory of Coulomb blockade or quasi one-dimensional variable range hopping conductivity with variable length of hops between localized states one-dimensional hopping conductivity or Coulomb gap conductivity [19]. Such changes occur due to changes of current carries density on the Fermi level due to interaction with bromine.

Possible Mechanism of MWNT Bromination. Decrease of the electrical conductivity is related with the bromination mechanism which can be described in the following way. Initial MWNTs possess structural defects on their surface-such as holes in graphene layers and nanosized curved graphitic sheets. Such structural defects have higher concentration of carboxylic groups and higher reactivity as compared with intact inner walls of nanotubes and react with bromine vapors according to following general reaction:

$$
\text { MWNT-COOH }+\mathrm{Br}_{2} \longrightarrow \text { MWNT-COO-Br }+\mathrm{HBr}
$$

Hydrogen bromide can further react with unsaturated bonds situated on defect areas of nanotubes:

$$
\text { MWNT- } \mathrm{CH}=\mathrm{CH}_{2}+\mathrm{HBr} \longrightarrow \text { MWNT- } \mathrm{CHBr}-\mathrm{CH}_{3}
$$

Bromination of annealed nanotubes results in decrease of the Br content on the surface of MWNTs as determined by XPS: 0.15 at. $\%$ for annealed nanotubes versus 0.5 at. $\%$ for untreated nanotubes (Figure 5(a)). The reason for this may be lower amount of structural and topological defects on the surface of annealed sample. Perfect graphene layers, that is, nanotube walls, are less able to react with bromine vapors at moderate temperatures. The same behavior is known for single-wall carbon nanotubes which have more perfect structure than multiwall nanotubes. In the case of absence of "dangling bonds," curved graphitic layers or holes in the 
nanotube walls, the reaction between halogen molecule and the surface of nanotube is strongly impeded. Formation of covalent bond between halogen and carbon layer proceeds with partial transition of electron density to the halogen from conjugated $\pi$-system of carbon atoms with subsequent change of the hybridization state of carbon from $s p^{2}$ to $s p^{3}$. This process is energetically unfavorable in the case of perfect structure of brominated objects-that is, single-wall nanotubes or graphitized annealed nanotubes.

Another way of interaction of pristine MWNTs with bromine vapors is formation of "intercalate-like" structures with $\mathrm{Br}$ atoms between tangled graphene layers or $\mathrm{Br}$ chemisorption on the defective areas of nanotubes (Figure 5(b)). Both these types of linked $\mathrm{Br}$ atoms are seen on the high-resolution XPS spectrum for the sample MWNT ${ }^{\mathrm{Br}}$. Low-defective annealed MWNT sample shows lower amount of total bonded bromine and higher content of covalently linked $\mathrm{Br}$ atoms. Thus it allows one to conclude that the bromination of MWNTs proceeds via interaction with surface defects and functional groups of carbon nanotubes.

\section{Conclusions}

Vapor-phase reaction with bromine vapors at moderate temperature was successfully used for dry synthesis of $\mathrm{Br}$ functionalized multiwall carbon nanotubes with overall bromine content $\sim 2.5 \mathrm{wt}$. \%. Bromine is covalently bonded to the surface of the nanotube and no structural changes are observed with XPS, TEM, and SEM methods. Amount of Brcontaining groups corresponds to that for carboxylic groups of initial nanotubes, thus possible mechanism of bromination can be formation of bromoanhydride-like functional groups.

Investigation of electrophysical properties of brominated nanotubes reveals significant changes in the conductivity due to interaction of $\mathrm{Br}$ atoms with the surface of the nanotube and consecutive changes in the current carries density on the Fermi level.

Br-functionalized carbon nanotubes can be further used for design of tailor-made materials using approaches of organometallic synthesis.

\section{Acknowledgments}

This work was supported by RFBR Grant no. 11-03-00351, ISTC Project no. 1708-B and Russian FASI Projects no. 16.740.11.0016, and no. 16.740.11.0146.

\section{References}

[1] P.-C. Ma, N. A. Siddiqui, G. Marom, and J. K. Kim, "Dispersion and functionalization of carbon nanotubes for polymerbased nanocomposites: a review," Composites A, vol. 41, no. 10, pp. 1345-1367, 2010.

[2] J. K. Kim, Carbon Nanotubes for Polymer Reinforcement, CRC Press, 2011.

[3] J. F. Colomer, R. Marega, H. Traboulsi, M. Meneghetti, G. van Tendeloo, and D. Bonifazi, "Microwave-assisted bromination of double-walled carbon nanotubes," Chemistry of Materials, vol. 21, no. 20, pp. 4747-4749, 2009.
[4] J. F. Friedrich, S. Wettmarshausen, S. Hanelt et al., "Plasmachemical bromination of graphitic materials and its use for subsequent functionalization and grafting of organic molecules," Carbon, vol. 48, no. 13, pp. 3884-3894, 2010.

[5] S. C. Ray, C. W. Pao, H. M. Tsai et al., "Electronic structures and bonding properties of chlorine-treated nitrogenated carbon nanotubes: X-ray absorption and scanning photoelectron microscopy studies," Applied Physics Letters, vol. 90, no. 19, Article ID 192107, 2007.

[6] V. A. Volodina, A. A. Kozlovskii, S. I. Kuzina, and A. I. Mikhailov, "Direct and initiated halogenation of carbon nanomaterials at low temperatures," High Energy Chemistry, vol. 42, no. 4, pp. 311-318, 2008.

[7] A. Diacon, E. Rusen, B. Mărculescu et al., "Superficial grafting of water-soluble polymers on brominated mwcnt by atrp technique," International Journal of Polymer Analysis and Characterization, vol. 16, no. 1, pp. 1-8, 2011.

[8] G. Fanchini, H. E. Unalan, and M. Chhowalla, "Modification of transparent and conducting single wall carbon nanotube thin films via bromine functionalization," Applied Physics Letters, vol. 90, no. 9, Article ID 092114, 2007.

[9] Y. K. Chen, M. L. H. Green, J. L. Griffin, J. Hammer, R. M. Logo, and S. C. Tsang, "Purification and opening of carbon nanotubes via bromination," Advanced Materials, vol. 8, no. 12, pp. 1012-1015, 1996.

[10] J. Li, L. Vaisman, G. Marom, and J. K. Kim, "Br treated graphite nanoplatelets for improved electrical conductivity of polymer composites," Carbon, vol. 45, no. 4, pp. 744-750, 2007.

[11] E. Papirer, R. Lacroix, J. B. Donnet, G. Nanse, and P. Fioux, "XPS Study of the halogenation of carbon black-part 1 . Bromination," Carbon, vol. 32, no. 7, pp. 1341-1358, 1994.

[12] E. Unger, A. Graham, F. Kreupl, M. Liebau, and W. Hoenlein, "Electrochemical functionalization of multi-walled carbon nanotubes for solvation and purification," Current Applied Physics, vol. 2, no. 1, pp. 107-111, 2002.

[13] V. L. Kuznetsov, K. V. Elumeeva, A. V. Ishchenko et al., "Multiwalled carbon nanotubes with ppm level of impurities," Physica Status Solidi B, vol. 247, no. 11-12, pp. 2695-2699, 2010.

[14] S. Doniach and M. Sunjic, "Many-electron singularity in X-ray photoemission and X-ray line spectra from metals," Journal of Physics C, vol. 3, no. 2, pp. 285-291, 1970.

[15] D. A. Shirley, "High-resolution X-ray photoemission spectrum of the valence bands of gold," Physical Review B, vol. 5, no. 12, pp. 4709-4714, 1972.

[16] C. Fitzwater, Functionalization of multi-walled carbon nanotubes in epoxy composites, M.S. thesis, 2010.

[17] A. I. Romanenko, O. B. Anikeeva, T. I. Buryakov et al., "Influence of surface layer conditions of multiwall carbon nanotubes on their electrophysical properties," Diamond and Related Materials, vol. 19, no. 7-9, pp. 964-967, 2010.

[18] B. L. Altshuler and A. G. Aronov, "Electron-electron interaction in disordered conductors," in Electron-Electron Indisordered Systems, A. L. Efros and M. Pollak, Eds., North-Holland, 1985.

[19] Z. R. Ismagilov, A. E. Shalagina, O. Y. Podyacheva et al., "Structure and electrical conductivity of nitrogen-doped carbon nanofibers," Carbon, vol. 47, no. 8, pp. 1922-1929, 2009. 

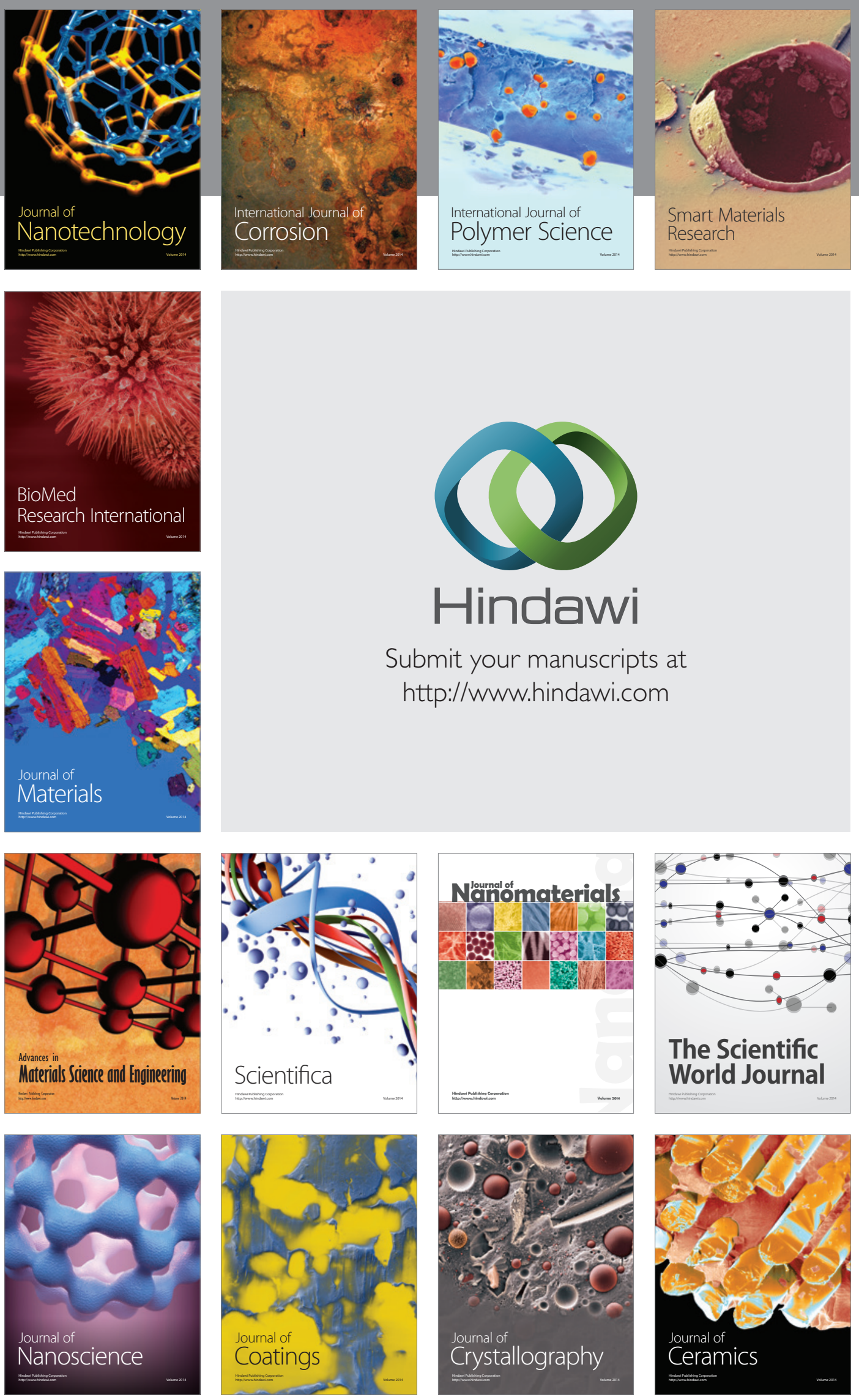

The Scientific World Journal

Submit your manuscripts at

http://www.hindawi.com

\section{World Journal}

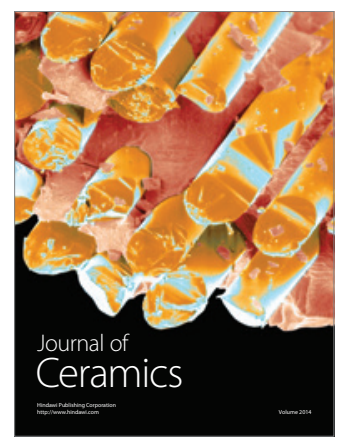

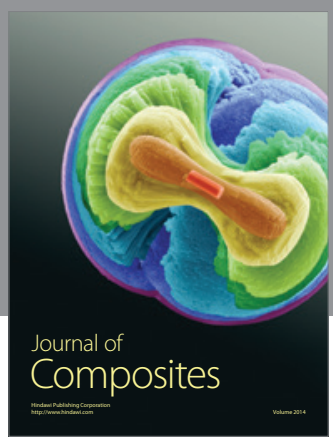
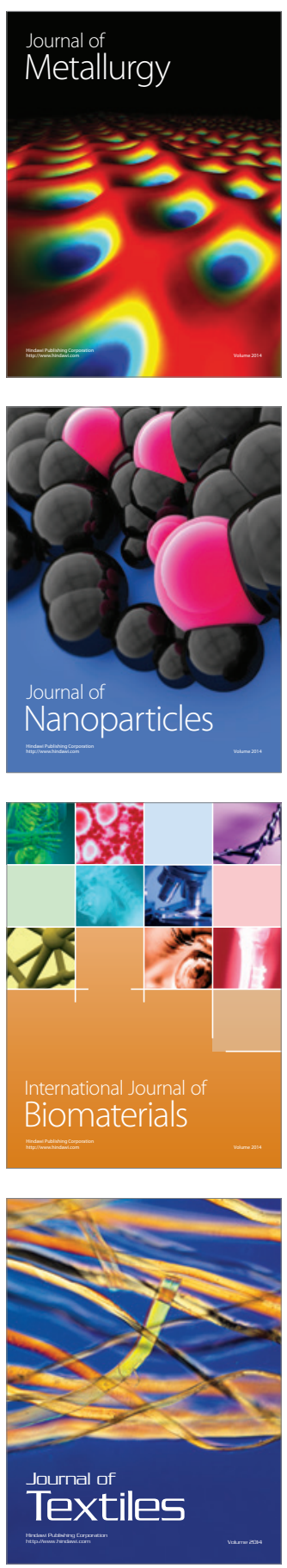